\title{
Evaluación de la adhesión de sistemas adhesivos de grabado total en esmalte dental bovino usando un agente desproteinizante: un estudio in vitro.
}

\author{
Evaluation of adhesion of total etch adhesive systems on bovine \\ dental enamel using a deproteinizing agent: an in vitro study.
}

\author{
Michelle Gerardo Lang-Salas, ${ }^{*}$ Luz Alejandra Villarreal-Romero, ${ }^{\ddagger}$ Jessica Anaí Domínguez-Monreal, ${ }^{\ddagger}$ \\ Juan Carlos Cuevas-González, ${ }^{*}$ Alejandro Donohué-Cornejo, ${ }^{*}$ Simón Yobanny Reyes-López, \\ Erasto Armando Zaragoza-Contreras, ${ }^{\S}$ León Francisco Espinosa-Cristóbal*
}

\section{RESUMEN}

Introducción: Hoy en día, la mayoría de los pacientes requieren tratamiento de ortodoncia fija convencional, la cual utiliza procedimientos adhesivos afines con la superficie amelodentinaria. Aunque existen diferentes técnicas adhesivas que pueden ofrecer adecuados niveles de resistencia al desalojo, el uso del hipoclorito de sodio $(\mathrm{NaClO})$ como agente desproteinizante no ha sido bien definido en materiales de cementación en aparatología ortodóncica convencional. Objetivo: El objetivo de este estudio fue determinar los niveles de resistencia al desalojo con pruebas de microtensión y análisis topográfico con microscopia electrónica de barrido. Material y métodos: 20 bloques de esmalte bovino fueron expuestos superficialmente a soluciones acondicionadoras (ácido ortofosfórico al 37\%) con y sin agente desproteinizante ( $\mathrm{NaClO}$ al $5.25 \%$ ); finalmente, brackets individuales de ortodoncia fueron cementados con sistemas adhesivos y resinas compuestas de casas comerciales (Ormco y 3M). Las pruebas de resistencia al desalojo fueron realizadas con una máquina de fuerzas universales y el análisis topográfico con microscopia electrónica de barrido. Resultados: $\mathrm{El}$ uso del $\mathrm{NaClO}$ al $5.25 \%$ y la marca Ormco tuvieron estadísticamente los mejores niveles de adhesión que el grupo sin desproteinización y la casa comercial 3M, respectivamente. La topografía adamantina expuesta al agente desproteinizante mostró características de superficie relacionadas con el patrón de grabado tipo I. Conclusión: El uso de un agente desproteinizante ( $\mathrm{NaClO}$ $5.25 \%$ ) así como una marca comercial (Ormco) en particular podrían definir el aumento en los niveles de adhesión en superficies adamantinas con aparatología ortodóncica convencional.

Palabras clave: Sistemas adhesivos, ortodoncia, agente desproteinizante, bracket.

\section{ABSTRACT}

Introduction: Nowadays, patients require conventional fixed orthodontic treatment, which uses adhesive procedures related to the dentinal denture surface. Although there are different adhesive techniques that can offer adequate levels of bonding resistance, the use of $\mathrm{NaClO}$ as a deproteinizing agent has not been well defined in cementing materials in conventional orthodontic appliances. Objective: The objective of this study was to determine the levels of bond resistance using micro tension tests and topographic analysis with scanning electron microscopy. Material and methods: Twenty-blocks of bovine enamel were exposed superficially to conditioning solutions (37\% orthophosphoric acid) with and without deproteinizing agent $(5.25 \% \mathrm{NaClO})$; finally, individual orthodontic brackets were cemented with adhesive systems and resin composites of different trademarks (Ormco and 3M). Bonding resistance tests were performed with a universal force machine and topographic analysis was made with scanning electron microscopy. Results: The use of $5.25 \% \mathrm{NaClO}$ and the Ormco brand had statistically better levels of adhesion than the group without deproteinization and the $3 M$ brand, respectively. Adamantine topography exposed to the deproteinizing agent showed surface characteristics related to the type I etching pattern. Conclusion: The use of a deproteinizing agent ( $\mathrm{NaClO} \mathrm{5.25 \% )}$ as well as a trademark (Ormco) in particular conditions could define the increase in adhesion levels on adamantine surfaces with conventional orthodontic appliances.

Keywords: Adhesive systems, orthodontics, deproteinizing agent, bracket.

\footnotetext{
* Maestría en Ciencias Odontológicas, Departamento de Estomatología, Instituto de Ciencias Biomédicas, Universidad Autónoma de Ciudad Juárez (UACJ), Ciudad Juárez, Chihuahua.

‡ Especialidad de Ortodoncia, Departamento de Estomatología, Instituto de Ciencias Biomédicas, Universidad Autónoma de Ciudad Juárez (UACJ), Ciudad Juárez, Chihuahua.

§ Laboratorio de Polímeros, Centro de Investigación en Materiales Avanzados (CIMAV), Chihuahua, Chihuahua.
}

Recibido: 01 Octubre 2019. Aceptado para publicación: 18 Noviembre 2019.

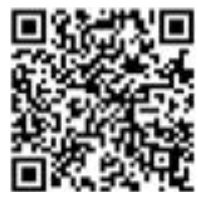




\section{INTRODUCCIÓN}

L a salud oral integral es la meta de la odontología, de los cuales uno de los principales problemas es la malposición de los órganos dentales, que afectan tanto la función como la estética, y la manera más adecuada de corregirlo es la ortodoncia. ${ }^{1}$ La adhesión es el resultado de un conjunto de interacciones que contribuyen a unir dos superficies, el esmalte por un lado y la base del bracket por otro; esta unión se produce por medio de un cemento. ${ }^{2}$ Los dos tipos de adhesión que pueden producirse en ortodoncia son la adhesión mecánica, la cual se realiza por la penetración del material de cementado en las rugosidades de la superficie, y la adhesión química, que corresponde a la unión íntima, a escala molecular entre la base y el adhesivo. En este sentido, se debe establecer que la adhesión en los tratamientos ortodóncicos debería ser un proceso reversible que no deje daños estructurales en el tejido adamantino una vez finalizado el tratamiento. ${ }^{2}$

En la actualidad se utilizan distintos sistemas adhesivos para cementar los brackets al esmalte dental. Un adhesivo es una sustancia capaz de mantener adheridos dos materiales por unión superficial, los sistemas adhesivos han evolucionado a lo largo de los años, son el resultado de investigaciones dedicadas a buscar mayor biocompatibilidad, con una adecuada resistencia al desalojo y sin dañar el esmalte dental. Sin embargo, la principal preocupación al emplear estos métodos de adhesión es por la falla en la unión a la estructura dental. ${ }^{3}$ Hoy en día, el bracket ortodóncico es considerado un dispositivo, metálico o cerámico, que define los movimientos ortodóncicos procedentes de la aplicación de una fuerza. El desalojo de un bracket trae consigo pérdida de tiempo en la consulta, retraso en la evolución del tratamiento y pérdida del esmalte superficial rico en flúor. Los fracasos en la adhesión se dan por varios factores: mala técnica del operador, variaciones de la superficie del esmalte, contaminación por saliva, malos hábitos del paciente, brackets de mala calidad y las fuerzas de masticación. ${ }^{4}$ Una restauración dental a base de resina debe durar adherida el mayor tiempo posible; sin embargo, la aparatología ortodóncica debería durar adherida aproximadamente dos años, y debería poder ser desalojada con una fuerza de entre 5.9 a $7.8 \mathrm{MPa}^{5}$

En los últimos años se ha popularizado el uso del hipoclorito de sodio $(\mathrm{NaClO})$ como agente desproteinizante previo al grabado ácido, ya que ha demostrado aumentar la superficie retentiva del esmalte; sin embargo, faltan estudios para comprobar su efectividad. ${ }^{6}$ El hipoclorito de sodio en una concentración de 5.25\% actúa como agente bactericida y bacteriostático eliminando las proteínas desnaturalizadas por medio de un proceso de desproteinización, lo cual genera la aparición de canales tridimensionales y logra que un agente adhesivo sea capaz de quedar retenido, dando lugar a una adhesión más óptima. ${ }^{7}$ Aunque existen diversos estudios que han evaluado el mejoramiento de las propiedades adhesivas con el uso de agentes desproteinizantes en superficies adamantinas y dentinarias de seres humanos, existe información limitada de agentes cementantes usados ampliamente en la práctica ortodóncica que usen agentes desproteinizantes previo al grabado total convencional en muestras de esmalte bovino. ${ }^{1} \mathrm{El}$ esmalte bovino ha sido ampliamente utilizado en procedimientos de adhesión in vitro, debido a sus características estructurales adamantinas muy similares a la de los seres humanos. ${ }^{8}$ Lo anterior permite el desarrollo de pruebas mecánicas con un número de muestra más representativa, de fácil acceso, mejor área de trabajo, mejor control en la ética del estudio, entre otras ventajas. El objetivo de este estudio fue determinar el nivel de resistencia al desalojo de dos sistemas adhesivos de grabado total usados convencionalmente en tratamientos ortodóncicos expuestos a un agente desproteinizante en muestras de esmalte bovino.

\section{MATERIAL Y MÉTODOS}

\section{Recolección de la muestra}

A través de un estudio transversal comparativo in vitro se recolectaron 20 muestras de órganos dentarios anteriores bovinos, provenientes del rastro municipal de Ciudad Juárez, Chihuahua. Los dientes que se incluyeron fueron dientes anteroinferiores, excluyendo a todos aquéllos que mostraron algún tipo de alteración en la topografía adamantina. Una vez recolectados, todos los dientes fueron lavados a través de un baño ultrasónico por 15 minutos en agua desionizada a temperatura ambiente. El lavado se realizó en dos ciclos. Finalmente, las muestras fueron almacenadas en agua desionizada a $4{ }^{\circ} \mathrm{C}$.

\section{Preparación de las muestras}

Las coronas anatómicas fueron separadas de las porciones radiculares a través de un disco de diamante con irrigación a 3,000 rpm. Una vez separadas las coronas, las muestras fueron incluidas en bloques de resinas acrílicas aproximadamente de $2 \times 2 \mathrm{~cm}$, dejando libre la cara vestibular de las muestras. Las muestras de esmalte fueron aleatoriamente distribuidas en los siguientes grupos de estudio: 
a) Transbond XT, $3 \mathrm{M}$ con desproteinizante; b) Transbond $\mathrm{XT}, 3 \mathrm{M}$ sin desproteinizante; c) OrthoSolo ${ }^{\circledR}+$ Enlight $^{\circledR}$, Ormco con desproteinizante; d) OrthoSolo ${ }^{\circledR}+$ Enlight $^{\circledR}$, Ormco sin desproteinizante. Para los grupos sin el agente desproteinizante se utilizaron procedimientos recomendados para cada uno de los fabricantes. Para los grupos con el agente desproteinizante se utilizó una solución de $\mathrm{NaClO}$ al $5.25 \%$ aplicado por 60 segundos con un microcepillo a las superficies adamantinas, posteriormente las superficies fueron lavadas con agua desionizada por 30 segundos; finalmente, cada sistema adhesivo fue colocado de acuerdo con las recomendaciones de cada fabricante.

\section{Ensayo de resistencia al desalojo}

Para determinar el nivel de adhesión de los sistemas adhesivos a la estructura adamantina se utilizaron pruebas de microtensión a través de una máquina de fuerzas universal y se utilizó una velocidad de desalojo de 0.5 $\mathrm{mm} / \mathrm{min}$ en sentido inciso-apical.

\section{Análisis con microscopia electrónica de barrido (MEB)}

El análisis topográfico y químico de las muestras de esmalte fueron realizadas con un microscopio electrónico de barrido (Hitachi ${ }^{\odot}$, FE-SEM, SU5000) con el uso de detectores de electrones secundarios y electrones retrodispersados con una intensidad de voltaje de $15 \mathrm{kV}$.

\section{Análisis estadístico}

Los valores de resistencia al desalojo fueron expresados en promedio y desviación estándar con el programa IBM-SPSS v23 y las gráficas fueron generadas con el programa Origin.

\section{RESULTADOS}

\section{Pruebas de resistencia al desalojo}

En la Figura 1 se demuestran los resultados de las pruebas de microtensión. Se puede observar que el grupo con el agente desproteinizante obtuvo significativamente valores más elevados (12.8 $\pm 4.5 \mathrm{MPa}$ ) comparado con las muestras sin desproteinizante $(6.0 \pm 1.8 \mathrm{MPa})$ (Figura 1A). Además, el agente cementante de la marca ORMCO también demostró estadísticamente valores más incrementados $(11.2 \pm 4.4 \mathrm{MPa}$ ) que la marca $3 \mathrm{M}(7.6 \pm 4.8 \mathrm{MPa})$ (Figura 1B). Por otro lado, cuando se realizaron las comparaciones independientes entre subgrupos otras diferencias estadísticamente significativas fueron encontradas. En los grupos no desproteinizados, la marca ORMCO demostró valores de resistencia al desalojo significativamente mejores (7.6 \pm $5.0 \mathrm{MPa}$ ) que el grupo de $3 \mathrm{M}(4.5 \pm 4.4 \mathrm{MPa})$ (Figura 1C); sin embargo, el uso del agente desproteinizante mejoró en general los valores de resistencia (ORMCO $=14.8 \pm$ 7.2 $\mathrm{MPa}$ y $3 \mathrm{M}=10.8 \pm 4.3 \mathrm{MPa}$ ), ninguna diferencia
A) 20

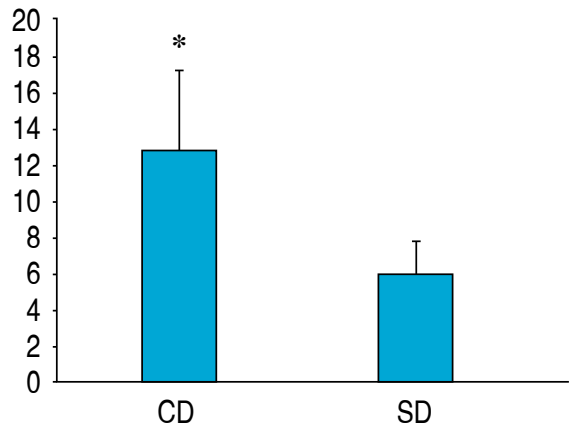

Figura 1:

Ensayos de microtensión.

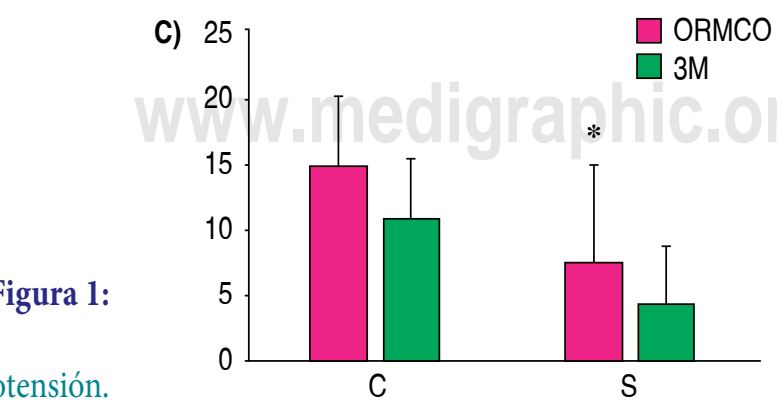

B) 18

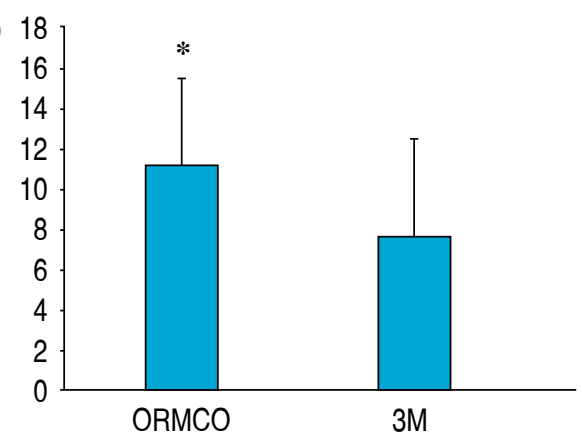

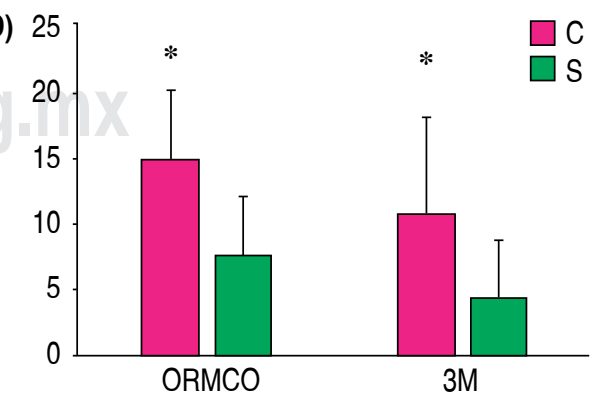




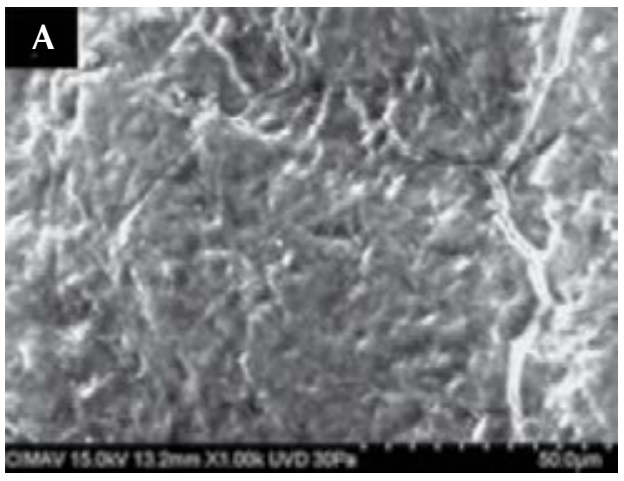

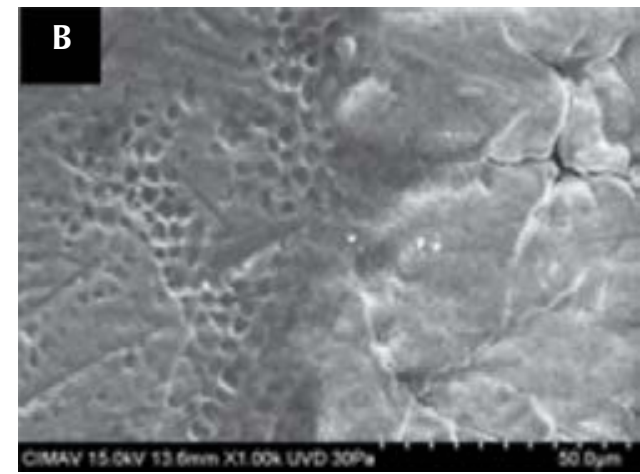

Figura 2:

Micrografía por MEB. A) Sin desproteinización, x1,000; B) Con desproteinización, x1,000. estadística fue identificada cuando se compararon ambas marcas comerciales (Figura 1D).

\section{Microscopia electrónica de barrido}

En la Figura 2 se observan micrografías con MEB de muestras desproteinizadas y sin desproteinizar. La Figura $2 \mathrm{~A}$ muestra aparentemente superficies obturadas con presencia de elementos que dificultan la observación directa de los prismas adamantinos. Por otro lado, la Figura $2 B$ exhibe relativamente superficies adamantinas más despejadas en las que la visualización de los prismas adamantinos es más clara y limpia, probablemente expresando patrones de grabado tipo I.

\section{DISCUSIÓN}

Este estudio demostró que el uso de un agente desproteinizante a base de hipoclorito de sodio mejoró el nivel de resistencia al desalojo en muestras de forma in vitro, particularmente en aquellas muestras en las que la marca Ormco como agente cementante estuvo presente. Aunque se encontraron diferencias estadísticamente significativas entre las marcas comerciales usadas como cementantes, la marca $3 \mathrm{M}$ también mostró mejoras en los valores de adhesión cuando el agente desproteinizante fue utilizado. Esto indica que el uso del agente desproteinizante en esmalte dental sano (usado en nuestro estudio) mejora las características de adhesión, incluso en agentes cementantes que ofrecen propiedades de resistencia al desalojo más disminuidas.

Un estudio evaluó $\mathrm{NaClO}$ al $5.25 \%$ en esmalte dental sano, utilizó prismas de resina y encontró que el uso de un agente desproteinizante mejoró los niveles de resistencia al desalojo (58.41 $\mathrm{MPa}$ ) comparado con el acondicionamiento tradicional (40.1 MPa); además, las fallas cohesivas fueron más frecuentes con el agente desproteinizante (85\%) que con el acondicionamiento convencional (15\%). Estos autores concluyeron que la desproteinización del esmalte previo al grabado ácido es fundamental para lograr una mayor adhesión entre el esmalte y la resina. ${ }^{1}$ Otro estudio realizado en 2017 determinó que el hipoclorito de sodio al 5.25\% por un minuto obtuvo mayores resistencias al desprendimiento de brackets, concluyendo que el uso de un agente desproteinizante tiene la habilidad de eliminar la capa proteica de las superficies adamantinas. ${ }^{9}$ Otros estudios también han concluido que el uso de $\mathrm{NaClO}$ como agente desproteinizante podría mejorar la durabilidad de diferentes tipos de restauraciones en esmalte o dentina por tiempos más prolongados. ${ }^{10,11}$ Por otro lado, se ha reportado que el sistema adhesivo OrthoSolo seguido de la resina compuesta Enlight, ambos de la casa comercial ORMCO, pueden ofrecer valores de resistencia al desalojo adecuados para tratamientos ortodóncicos que van de los 9.79 a los $20.83 \mathrm{MPa},{ }^{12}$ mientras que el sistema adhesivo Transbond XT de la casa comercial $3 \mathrm{M}$ ofrece valores de adhesión alrededor de $10.23 \mathrm{MPa} .^{13}$ Nuestro estudio encontró que el uso de un agente desproteinizante durante 60 segundos previo al grabado ácido aumenta la adhesión en esmalte $(p<0.05)$. Al comparar los sistemas adhesivos se obtuvieron resultados estadísticamente significativos que demostraron que la resina de ORMCO tenía una mayor fuerza de unión al esmalte que la de $3 \mathrm{M}$ (Figura 1B), pero también, nuestros resultados indicaron que la presencia del agente desproteinizante y la marca $3 \mathrm{M}$ tienden a incrementar los valores de resistencia al desalojo. (Figuras 1 C y D). Lo anterior indica que el uso de un agente desproteinizante y un tipo de sistema en particular, como lo es Ormco, podrían intervenir en el mejoramiento de la resistencia al desalojo.

Por otro lado, diversos estudios han analizado la tipografía obtenida con el uso de $\mathrm{NaClO}$ como agente desproteinizante a través de MEB. Estudios determinaron 
que el $\mathrm{NaClO}$ al 5-5.25\% (15-60 s) usado previamente al acondicionamiento total con ácido ortofosfórico al $37 \%$ (15 s) genera estadísticamente mejores niveles de adhesión y el aumento en la frecuencia de patrones de grabado I y II comparado con aquellas técnicas que no usan el agente desproteinizante $(p<0.05) \cdot{ }^{14,15}$ Otro estudio determinó variaciones topográficas en superficies adamantinas expuestas al $\mathrm{NaClO}$ previo al grabado ácido, determinando que el uso del agente desproteinizante ( $\mathrm{NaClO}$ al $2.5 \%$ ), previo al grabado ácido, presenta estructuras prismáticas no uniformes e irregulares sugiriendo la presencia de patrones de acondicionamiento adamantino tipo I y II, los cuales podrían mejorar las características adhesivas entre la superficie amelodentinaria con los sistemas adhesivos. ${ }^{16}$ Nuestros resultados coinciden con los previamente publicados en los que el uso de un agente desproteinizante como el $\mathrm{NaClO}$ al 5.25\%, previo al grabado ácido, genera topografías relacionadas a los patrones de grabado tipo I, el cual se representa con socavados internos e irregulares del prisma adamantino. ${ }^{15}$ Lo anterior sugiere que el $\mathrm{NaClO}$ previo al grabado ácido convencional podría facilitar la formación de una topografía de la superficie adamantina con mejores características para el incremento de la adhesión a través de la formación de capas híbridas más adecuadas. ${ }^{17,18}$ Aunque este estudio podría apoyar el uso del $\mathrm{NaClO}$ al 5.25\%, previo al grabado ácido convencional para el mejoramiento de la adhesión en la aparatología ortodóncica, es necesario realizar otros estudios que involucren muestras biológicas tomadas directamente de pacientes, analizando otras variables que influyan en el desarrollo de mejores y adecuadas capas híbridas a través del uso de ambientes orales simulados.

\section{CONCLUSIONES}

Este estudio demostró que el uso de un agente desproteinizante como el $\mathrm{NaClO}$ al $5.25 \%$ puede incrementar significativamente los niveles de adhesión en esmalte dental bovino. Pero también, el uso de una marca comercial en específico, como lo es Ormco, puede intervenir en el mejoramiento de los niveles de adhesión. Aunque este estudio podría sugerir el uso del $\mathrm{NaClO}$ al 5.25\% para ayudar a mejorar los niveles de adhesión en sistemas adhesivos de grabado total usados con frecuencia en la cementación de aparatología ortodóncica, es necesario incluir otros tipos de diseños experimentales que incluyan especímenes biológicos o artificiales que simulen las características estructurales de los órganos dentales humanos.

\section{AGRADECIMIENTOS}

Los autores agradecen al Consejo Nacional de Ciencia y Tecnología (CONACYT), al Programa para el Desarrollo Profesional Docente (PRODEP) y al Departamento de Estomatología de la Universidad Autónoma de Ciudad Juárez por el apoyo en la realización de este trabajo.

\section{BIBLIOGRAFÍA}

1. Espinosa R, Valencia R, Rabelero M, Ceja I. Resistencia al desprendimiento de la resina al esmalte desproteinizado y grabado; estudio de microtensión detachment resistance to resin and deprotenized and etch enamel. [Accessed September 9, 2019] http://www.rodyb.com/resistencia-microtension/.

2. Dallel I, Lahwar S, Jerbi MA, Tobji S, Ben Amor A, Kassab A. Impact of adhesive system generation and light curing units on orthodontic bonding: in vitro study. Int Orthod. August 2019. doi:10.1016/j. ortho.2019.08.020

3. Salazar MP, Zamarripa DE, Tinoco CV, Oliver PR. Resistencia al desalojo de brackets metálicos expuestos a alimentos ácidos. Ortodon Actual. 2013; 9 (37): 14-22.

4. Aguilar E, Ferreto Gl, Rodríguez WL, Cáceres ZH. Bond strength of an orthodontic adhesive system applied at several time intervals. Publicación Científica Facultad de Odontología. UCR | No.152013, ISSN: 1659-1046.

5. Newman GV, Newman RA, Sengupta AK. Comparative assessment of light-cured resin-modified glass ionomer and composite resin adhesives: in vitro study of a new adhesive system. Am J Orthod Dentofac Orthop. 2001; 119 (3): 256-262. doi:10.1067/ mod.2001.111552 (sin doi).

6. Ahuja B, Yeluri R, Sudhindra Baliga M, Munshi AK. Enamel deproteinization before acid etching-A scanning electron microscopic observation. J Clin Pediatr Dent. 2010. doi: 10.17796/ jcpd.35.2.9gw7147381836380.

7. Zaragoza MM, Sánchez FA, Ramírez CT, Ramos AM. Comparación de diferentes soluciones antimicrobianas en la desinfección del respaldo del sillón dental. Odont Act. 2014; 11 (137): 4-12. [Accessed September 11, 2019] https://www.researchgate.net/ publication/273692835_Comparacion_de_diferentes_soluciones antimicrobianas_en_la_desinfeccion_del_respaldo_del_sillon_ dental.

8. Posada MC, Sánches CF, Gallego GJ, Peláez VA, Restrepo LF, López JD. Dientes de bovino como sustituto de dientes humanos para su uso en la odontología. Revisión de literatura. Revista CES Odontología. 2006; 19 (1): 63-68. [Accessed September 9, 2019] https://www.researchgate.net/publication/277191205_Dientes_ de_bovino_como_sustituto_de_dientes_humanos_para_su_uso_ en_la_odontologia_Revision_de_literatura.

9. Erazo Montenegro $\overline{M E}$, Evelin $\bar{M}$. Resistencia al desprendimiento de brackets mediante fuerzas de cizallamiento, en el esmalte dental previamente desproteinizado. Estudio in vitro en la Facultad de Odontología de la Universidad Central del Ecuador. 2017. [Accessed March 25, 2019] http://www.dspace.uce.edu.ec/ handle/25000/8318.

10. Hamdane N, Kmeid R, Khoury E, Ghoubril J. Effect of sandblasting and enamel deproteinization on shear bond strength of resinmodified glass ionomer. Int Orthod. 2017; 15 (4): 600-609. doi:10.1016/j.ortho.2017.09.005. 
11. Siqueira F, Cardenas A, Gomes GM, Chibinski AC, Gomes O et al. Three-year effects of deproteinization on the in vitro durability of resin/dentin-eroded interfaces. Oper Dent. 43 (1): 60-70. doi:10.2341/16-308-L.

12. Godard M, Deuve B, Lopez I, Hippolyte MP, Barthélemi S. Shear bond strength of two 2-step etch-and-rinse adhesives when bonding ceramic brackets to bovine enamel. Int Orthod. 2017; 15 (3): 388404. doi:10.1016/j.ortho.2017.06.003.

13. Twomley J, Yu Q, Ballard R, Armbruster P, Xu X. Formulation and characterization of antibacterial orthodontic adhesive. Dental Press J Orthod. 2019; 24 (4): 73-79. doi:10.1590/2177-6709.24.4.073079.oar.

14. López-Luján NA, Munayco-Pantoja ER, Torres-Ramos G, BlancoVictorio DJ, Siccha-Macassi A, López-Ramos RP. Deproteinization of primary enamel with sodium hypochlorite before phosphoric acid etching. Acta Odontol Latinoam. 2019; 32 (1): 29-35. [Accessed September 30, 2019] http://www.ncbi.nlm.nih.gov/ pubmed/31206572.

15. Espinosa R, Valencia R, Uribe M, Ceja I, Saadia M. Enamel deproteinization and its effect on acid etching: an in vitro study. J Clin Pediatr Dent. 2008; 33 (1): 13-19. doi:10.17796/ jcpd.33.1.ng5462w5746j766p.

16. Abdelmegid FY. Effect of deproteinization before and after acid etching on the surface roughness of immature permanent enamel.
Niger J Clin Pract. 2018; 21 (5): 591-596. doi: 10.4103/njcp. njcp_166_17.

17. Hobson RS, Crotty T, Thomason JM, Jepson NJA. A quantitative study of enamel acid etch patterns on surfaces used for retention of resin-bonded fixed prostheses. Eur J Prosthodont Restor Dent. 2005; 13 (3): 123-128. [Accessed September 30, 2019] http:// www.ncbi.nlm.nih.gov/pubmed/16180638.

18. Hobson RS, Rugg-Gunn AJ, Booth TA. Acid-etch patterns on the buccal surface of human permanent teeth. Arch Oral Biol. 2002; 47 (5): 407-412. doi:10.1016/s0003-9969(02)00008-.

Correspondencia:

\section{Dr. En C. León Francisco Espinosa Cristóbal}

Maestría en Ciencias Odontológicas,

Departamento de Estomatología, Instituto de Ciencias Biomédicas,

Universidad Autónoma de Ciudad Juárez (UACJ), Anillo Envolvente del Pronaf s/n, Zona Pronaf, 32310, Ciudad Juárez, Chihuahua. Tel: (+52) 6566882100 ,

E-mail: leohamet@hotmail.com 